\title{
A pause for thought: exercise-induced sinus arrest causing syncope in a young male
}

\author{
John Whitaker, ${ }^{1}$ Matthew Wright, ${ }^{1,2}$ Mark $0^{\prime}$ Neill ${ }^{1,2}$ \\ 'Department of Cardiology, St Thomas' Hospital, London, UK; \\ 2King's College, London, UK
}

Correspondence to Dr John Whitaker, john.whitaker@trinity-oxford.com

\begin{abstract}
Summary
A 32-year-old Spanish man presented to hospital after a second episode of syncope immediately following exercise. On admission, his vitals signs were stable and he had a regular heart rate of $60 \mathrm{bpm}$. ECG and transthoracic echocardiogram were normal. He completed 15 min of a BRUCE protocol exercise test. One minute and ten seconds into recovery, he lost consciousness. His ECG demonstrated sinus arrest with pauses of up to $5 \mathrm{~s}$ and subsequently junctional ectopy. After $38 \mathrm{~s}$, his heart returned to sinus rhythm at a rate of $140 \mathrm{bpm}$ and he regained consciousness. Vasovagal syncope following exercise in the absence of structural heart disease is uncommonly reported. When cases of exercise-related syncope in patients with structurally normal hearts have been reported, the typical patient is a young male who engages in physical training. Treatment strategies in patients suffering with vasovagal asystole are necessarily empirical, and careful judgement based on the specific features of the individual cases needs to be employed.
\end{abstract}

\section{BACKGROUND}

Our case documents the presentation and investigation of a young man who experienced two syncopal episodes following exercise. Systematic investigation revealed an exercise-induced vasovagal cause of his syncope. The unusual nature of the diagnosis offers an opportunity to review the mechanism of vasovagal as well as exercise-induced syncope. This is relevant to both general medical specialists and junior doctors who are frequently called upon to investigate for and make a diagnosis of vasovagal syncope; however, the mechanism behind the process is seldom discussed.

\section{CASE PRESENTATION}

A 32-year-old Spanish man presented to hospital after a second episode of syncope immediately following exercise. On both occasions, he had exerted himself maximally for a short period. Within a minute of stopping, he described a sensation of light headedness lasting for several seconds and was aware of his vision fading. He regained consciousness on each occasion lying on the floor. On the second occasion, which had precipitated the admission to hospital, he had fallen backwards and sustained a minor laceration to the back of the head. He described no palpitations or chest pain and there were no features in the history suggestive of a seizure. The patient was previously well and kept physically fit, being currently in training to run a half marathon. He had no medical history of note and was on no regular medication. He described no family history of sudden cardiac death or arrhythmias. He had stopped smoking more than a year ago, used alcohol in moderation and denied using recreational drugs.

On admission, his vitals signs were stable and he had a regular heart rate of $60 \mathrm{bpm}$. Clinical examination was unremarkable. A baseline ECG confirmed sinus rhythm with a normal PR interval (124 ms) and narrow ORS complex
(81 ms) with a normal corrected QT interval (383.2 ms). There was minor ST elevation in lead V1 with minimal ST depression in lead III (figure 1). A transthoracic echocardiogram demonstrated normal biventricular function with no structural abnormality of his heart. He completed 15 min of a BRUCE protocol exercise test. His resting heart rate of $68 \mathrm{bpm}$ rose to a maximum heart rate of $187 \mathrm{bpm}$, representing $99 \%$ of the maximal, age-predicted heart rate. His resting blood pressure of $124 / 67 \mathrm{~mm} \mathrm{Hg}$ rose to a maximum of $169 / 75 \mathrm{~mm} \mathrm{Hg}$. He was asymptomatic throughout the exercise test. One minute and ten seconds into recovery, he described a feeling of dizziness and within $5 \mathrm{~s}$ had lost consciousness. His ECG demonstrated sinus arrest with a pause of $5 \mathrm{~s}$ (figure 2). The subsequent ECG strip demonstrated junctional ectopy, one beat of which was conducted retrogradely to the atrium and another junctional ectopic which was not (figure 3). He was placed in the recovery position and stimulation manoeuvres were attempted while resuscitation drugs were being prepared; however, after $38 \mathrm{~s}$ his heart returned to sinus rhythm at a rate of $140 \mathrm{bpm}$ and he regained consciousness describing a feeling of nausea but no other symptoms.

\section{INVESTIGATIONS}

12-Lead ECG: sinus rhythm with a normal PR interval (124 ms) and narrow ORS complex (81 ms) with a normal corrected QT interval (383.2 ms). There was minor ST elevation in lead V1 with minimal ST depression in lead III (figure 1).

Fifteen-minute BRUCE protocol exercise test: resting heart rate of $68 \mathrm{bpm}$ rose to a maximum heart rate of 187 bpm, representing $99 \%$ of the maximal, age-predicted heart rate. His resting blood pressure of $124 / 67 \mathrm{~mm} \mathrm{Hg}$ rose to a maximum of $169 / 75 \mathrm{~mm} \mathrm{Hg}$. He was asymptomatic throughout the exercise test. One minute and ten 


\section{BMJ Case Reports}

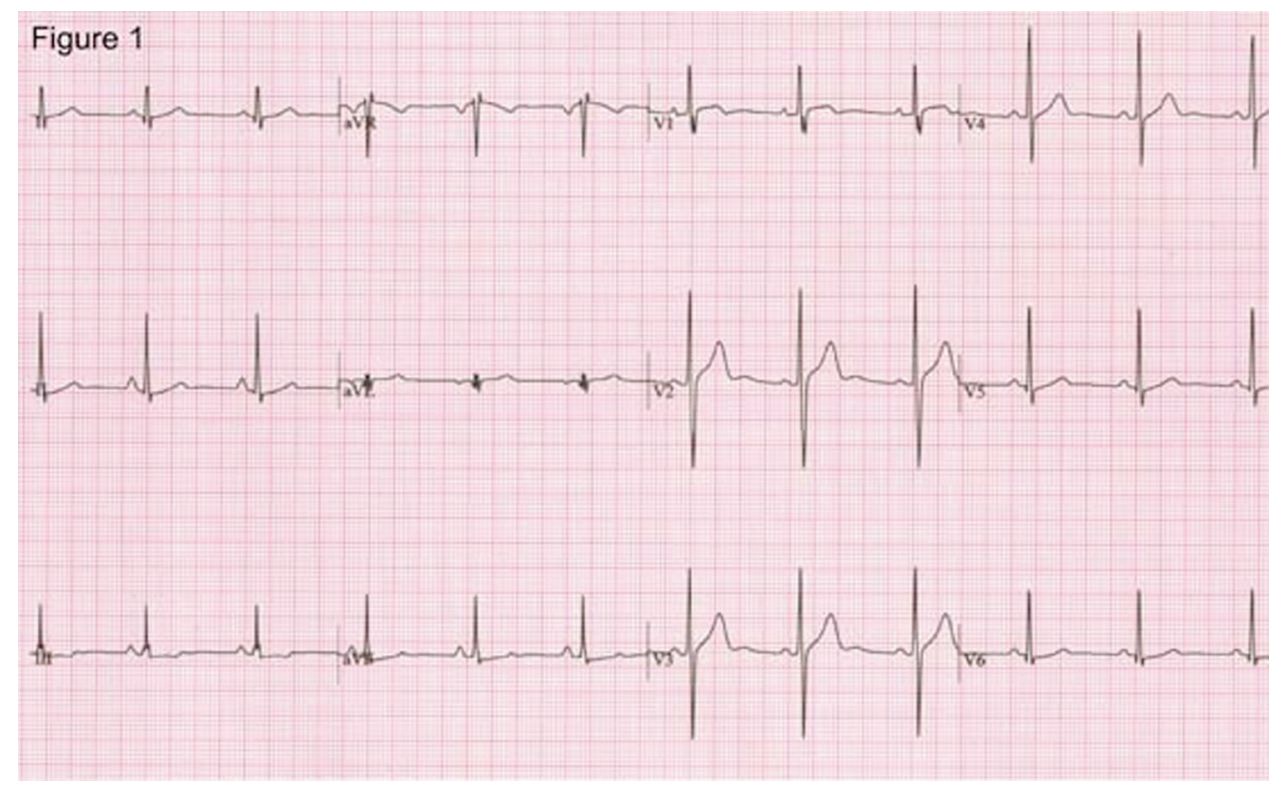

Figure 1 Resting 12-lead ECG showing sinus rhythm with a normal PR interval (124 ms) and narrow ORS complex (81 ms) with a normal corrected QT interval (383.2 ms), minor ST elevation in lead V1 with minimal ST depression in lead III.

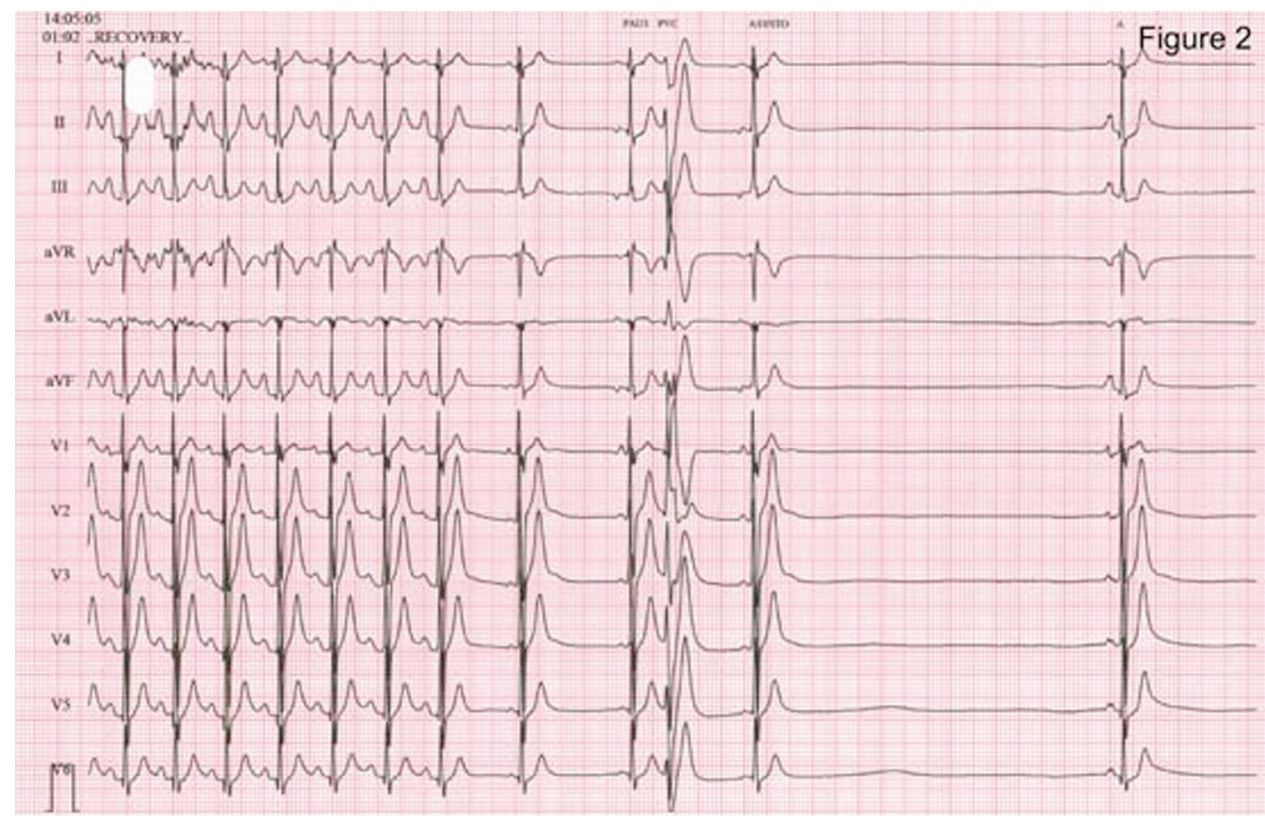

Figure 2 ECG taken 1 min and $10 \mathrm{~s}$ during recovery demonstrating sinus arrest with a pause of $5 \mathrm{~s}$.

seconds into recovery, he described a feeling of dizziness and within $5 \mathrm{~s}$ had lost consciousness. His ECG demonstrated sinus arrest with a pause of $5 \mathrm{~s}$ (figure 2).

The subsequent ECG strip demonstrated junctional ectopy, one beat of which was conducted retrogradely to the atrium and another junctional ectopic which was not (figure 3).

\section{TREATMENT}

As our patient did have a presyncopal aura and was wellinformed about his condition, it was felt appropriate to avoid pharmacotherapy or invasive devices and their risk of complications. Instead, strategies to avoid the potential complications of a syncopal episode were addressed. He was discharged with advice to maintain an adequate salt and fluid intake. He was educated as to the importance of taking evasive measures to maintain consciousness and to ensure his safety in the event of an impending syncopal attack.

\section{DISCUSSION}

The differential diagnosis for exercise-induced syncope is broad, encompassing structural heart disease (hypertrophic cardiomyopathy, critical aortic stenosis), coronary artery disease (myocardial infarction), arrhythmias (atrial fibrillation, supraventricular tachycardias and ventricular tachycardias - including both idiopathic and conditions predisposing to these rhythms such as Brugada syndrome, long QT syndrome and catecholaminergic polymorphic 


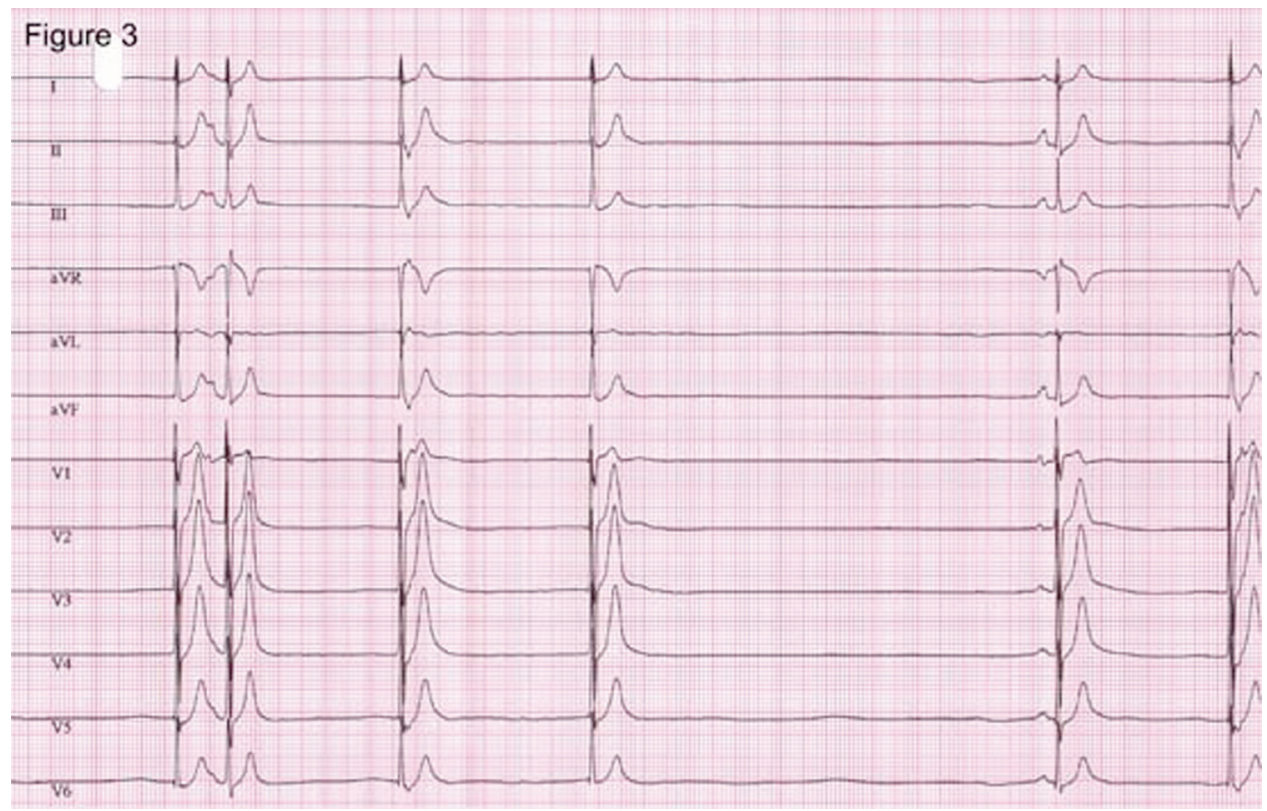

Figure 3 The subsequent ECG strip demonstrating junctional ectopy, with one beat conducted retrogradely to the atrium and another junctional ectopic which was not.

VT) and vasovagal syncope. Non-cardiac causes of syncope and psychogenic syncope have also been diagnosed, whereas $13-31 \%$ of patients presenting with syncope never reach a formal diagnosis. ${ }^{1}$ A thorough history is indicated as certain features have been shown to be associated with potentially life-threatening diagnosis of long QT syndrome. Where these features are present (which include a family history of syncope or sudden cardiac death, palpitations, and syncope associated with exercise and emotional stress), particular attention should be paid to the OT interval, ${ }^{2}$ which was of normal duration in our patient. In our patient, structural heart disease was excluded by transthoracic echocardiography. There were no features in the history to indicate coronary artery disease for which he had minimal risk factors. His exercise tolerance test demonstrated sinus arrest without any preceding arrhythmias consistent with a diagnosis of exercise-induced vasovagal syncope.

Vasovagal syncope is characterised by loss of consciousness resulting from cerebral hypoperfusion secondary to bradycardia and hypotension. ${ }^{3}$ Traditionally, vasovagal syncope has been explained by the Bezold-Jarisch reflex. Pooling of venous blood in the lower limbs (as can be induced diagnostically during tilt-table testing) results in decreased venous return to the heart and decreased ventricular filling. This triggers an increase in sympathetic tone causing vasoconstriction, increased force of ventricular contraction and increased heart rate; physiological mechanisms that allow the maintenance of blood pressure despite the decrease in venous return. The Bezold-Jarisch reflex occurs when, as a result of the sympathetically mediated increase in inotropy, low pressure baroreceptors located in the left ventricle respond to this increased tone by pathologically increasing their afferent input to the central nervous system resulting in decreased sympathetic drive and a profound vagal response. The result of this is bradycardia and hypotension responsible for the cerebral hypoperfusion causing syncope. ${ }^{3}$ It is now thought that this mechanism represents a single pathway of many potentially contributory mechanisms responsible for vasovagal syncope. Other mediators aside from the ventricular baroreceptors may include neurotransmitters such as serotonin, peripheral opioids and adenosine. ${ }^{3}$ Evidence for the presence of alternative mediators of vasovagal syncope comes from studies of cardiac transplant patients with denervated left ventricles who still experience vasovagal episodes. The hypothalamic and brainstem autonomic nuclei are likely to contribute also, perhaps particularly in those patients in whom vasovagal syncope is precipitated by emotion. ${ }^{4}$ Although it is accepted that changes in sympathetic tone are crucial to the vasodilatory response, the mechanisms through which this is mediated remain unclear. ${ }^{3}$

Vasovagal syncope following exercise in the absence of structural heart disease is uncommonly reported. ${ }^{5}$ Mechanisms involved are likely to include a parasympathetic response to the catecholamine release associated with exercise (with documented increase in heart rate and blood pressure), postexercise peripheral vasodilation with resultant decrease in venous return (mimicking the situation in tilt-table testing) as well as possibly augmented basal vagal tone seen in young athletes following physical training. ${ }^{6}$ When cases of exercise-related syncope in patients with structurally normal hearts have been reported, the typical patient is a young male who engages in physical training.

The long-term prognosis of exercise-induced vasovagal syncope is unclear. There is some evidence to suggest that vasovagal mechanisms may be contributory in survivors of presumed asystolic sudden death. ${ }^{7}$ However, vasovagal syncope is a very common condition and in the overwhelming majority of patients has a benign prognosis.

Successful treatment strategies for exercise-induced syncope have included exercise avoidance, increased salt and fluid intake, $\beta$-blockade, vagolytic agents and devices to protect against bradycardia. In the more general case of vasovagal syncope, trials have provided information that may be also relevant in the case of exercise-induced 


\section{BMJ Case Reports}

syncope. $\beta$-Blockers have not been demonstrated to be effective ${ }^{8}$ in preventing recurrence of syncope, whereas the evidence relating to pacemakers is more positive. A reduction in syncopal events has been reported in some trials, ${ }^{9}$ although the benefit of pacing would appear to be limited to those patients with a tilt positive cardioinhibitory syncope. ${ }^{10}$ As our patient did have a presyncopal aura and was well-informed about his condition, it was felt appropriate to avoid pharmacotherapy or invasive devices and their risk of complications. Instead, strategies to avoid the potential complications of a syncopal episode were addressed. He was discharged with advice to maintain an adequate salt and fluid intake. He was educated as to the importance of taking evasive measures to maintain consciousness and to ensure his safety in the event of an impending syncopal attack. Treatment strategies in patients suffering with vasovagal asystole are necessarily empirical, and careful judgement based on the specific features of the individual cases needs to be employed.

\section{Learning points}

- Vasovagal syncope is a common condition that is likely to be mediated by multiple mechanisms.

- A careful history may reveal features suggestive of long QT syndrome.

- Exercise-induced syncope requires systematic investigation to exclude structural heart disease, conduction system abnormalities and non-cardiac causes of syncope.

- Exercise-induced vasovagal syncope is rare but potentially dangerous.

- Treatment of exercise-induced vasovagal syncope is necessarily empirical, and the specific features of each case must be considered.
Competing interests None.

Patient consent Obtained.

\section{REFERENCES}

1. Miller TH, Kruse JE. Evaluation of syncope. Am Fam Physician 2005; 72:1492-500.

2. Colman N, Bakker A, Linzer $\mathrm{M}$, et al. Value of history-taking in syncope patients: in whom to suspect long QT syndrome? Europace 2009;11:937-43.

3. Fenton AM, Hammill SC, Rea RF, et al. Vasovagal syncope. Ann Intern Med 2000; 133:714-25.

4. Parry SW, Kenny RA. Tilt table testing in the diagnosis of unexplained syncope. OJM 1999;92:623-9.

5. Dockery BK, Newman KP. Exercise-induced asystole with syncope in a healthy young man. Am J Med Sci 2007;334:145-8.

6. Tse HF, Lau CP. Exercise-associated cardiac asystole in persons without structural heart disease. Chest 1995;107:572-6.

7. Milstein S, Buetikofer J, Lesser J, et al. Cardiac asystole: a manifestation of neurally mediated hypotension-bradycardia. J Am Coll Cardiol 1989;14:1626-32.

8. Sheldon R, Connolly S, Rose S, et al. Prevention of Syncope Trial (POST): a randomized, placebo-controlled study of metoprolol in the prevention of vasovagal syncope. Circulation 2006;113:1164-70.

9. Connolly SJ, Sheldon R, Roberts RS, et al. The North American Vasovagal Pacemaker Study (VPS). A randomized trial of permanent cardiac pacing for the prevention of vasovagal syncope. J Am Coll Cardiol 1999;33:16-20.

10. Sutton R, Brignole M, Menozzi C, et al. Dual-chamber pacing in the treatment of neurally mediated tilt-positive cardioinhibitory syncope: pacemaker versus no therapy: a multicenter randomized study. The Vasovagal Syncope International Study (VASIS) Investigators. Circulation 2000;102:294-9.

This pdf has been created automatically from the final edited text and images.

Copyright 2011 BMJ Publishing Group. All rights reserved. For permission to reuse any of this content visit http://group.bmj.com/group/rights-licensing/permissions.

BMJ Case Report Fellows may re-use this article for personal use and teaching without any further permission.

Please cite this article as follows (you will need to access the article online to obtain the date of publication).

Whitaker J, Wright M, O'Neill M. A pause for thought: exercise-induced sinus arrest causing syncope in a young male. BMJ Case Reports 2011;

10.1136/bcr.11.2010.3519, date of publication

Become a Fellow of BMJ Case Reports today and you can:

- Submit as many cases as you like

- Enjoy fast sympathetic peer review and rapid publication of accepted articles

- Access all the published articles

- Re-use any of the published material for personal use and teaching without further permission

For information on Institutional Fellowships contact consortiasales@bmjgroup.com

Visit casereports.bmj.com for more articles like this and to become a Fellow 